\title{
Liderazgo distribuido y empleo de las TIC para favorecerlo: un estudio de percepción en los IES de Melilla
}

\section{Distributed Leadership and the use of ICT to favor it: a perception study in the ISEs of Melilla}

\author{
Antonio Campos Soto ${ }^{1}$, Eduardo Chaves Barboza ${ }^{2}$, Esther Garzón Artacho ${ }^{3}$ \\ ${ }^{1}$ Universidad de Granada, España (acaso4@correo.ugr.es) \\ ${ }^{2}$ Universidad Nacional de Costa Rica, Costa Rica (echav@una.cr) \\ ${ }^{3}$ Universidad de Granada, España (esther.garzon.edu@juntadeandalucia.es)
}

Recibido el 14 de abril de 2017; revisado el 30 de agosto de 2017; aceptado el 2 de noviembre de 2017; publicado el 2 de diciembre de 2017

\section{RESUMEN:}

El artículo analiza el liderazgo distribuido en los institutos de educación secundaria (IES) de la Ciudad Autónoma de Melilla, y el empleo de las TIC para favorecer este liderazgo. El análisis se realiza a partir de las percepciones que presentan los órganos de gobierno y de coordinación didáctica de estos centros educativos.

Se ha utilizado una metodología cuantitativa, aplicando un cuestionario de 35 ítems, tipo escala ordinal, a una muestra de 145 sujetos $(\mathrm{N}=181)$. Sobre los datos se aplican técnicas de estadística descriptiva y análisis factoriales (análisis de componentes principales, con rotación Varimax).

Los resultados señalan que los equipos directivos distribuyen las funciones el liderazgo educativo, a pesar de que las TIC no se emplean directamente para implementar tales funciones.

PALABRAS CLAVE: LIDERAZGO, TIC, DOCENTE

\begin{abstract}
:
The article analyzes the distributed leadership on the institutes of secondary education (ISEs) of the Autonomous City of Melilla, and analyzed the use of ICT to improves this kind of leadership. The analysis is based on the perceptions of the governing and didactic coordination members of these educational centers.

A quantitative methodology was used, it has applied a questionnaire of 35 items, type ordinal scale, to a sample of 145 subjects $(\mathrm{N}=181)$. Descriptive statistics tests and factorial analysis (Principal Component Analysis with Varimax rotation) has been applied to the data.

The results indicate that management teams distribute the functions of educational leadership, even though ICTs are not directly used to implement such functions.
\end{abstract}

KEYWORDS:

LEADERSHIP, ICT, SECONDARY EDUCATION, TEACHING PRACTICE 


\section{INTRODUCCIÓN}

Resulta evidente que en el momento actual las tecnologías de la información y la comunicación (TIC) e Internet, junto con la globalización de la enseñanza, la continua actualización curricular y la necesidad de rendir cuentas, están propiciando un cambio metodológico y de interrelación en todos los sectores de la comunidad educativa de las escuelas y centros educativos, lo que redunda en un cambio a nivel organizativo, pedagógico y de gestión (Blau y Presser, 2013; Carreño, 2010; Cox, 2013; Dexter, 2011). Con el cambio de modelo de enseñanza, el liderazgo compartido puede fomentar comunidades de aprendizaje colaborativo en las que los profesores puedan trabajar juntos para integrar las TIC en su enseñanza y aprendizaje (Harris y Muijs, 2004; Schrum y Levin, 2012). De ahí que el modelo de liderazgo de los centros educativos afecte al currículo y la enseñanza de las TIC (White, 2014).

En España, con la reciente aprobación de la Ley Orgánica de Mejora de la Calidad Educativa (LOMCE), aunque cuestionada desde el principio por buena parte de la comunidad educativa, se han sentado las bases de una reforma que contribuirá a reforzar la capacidad de gestión de los centros; confiriendo a los directores, como representantes que son de la administración educativa (MECD, 2013) y como responsables del proyecto educativo, la oportunidad de ejercer un mayor liderazgo pedagógico y de gestión (MECyD, 2014). Al mismo tiempo, esta ley potencia la función directiva a través de un sistema de certificación para acceder al puesto de director, mediante la acreditación de una serie de competencias (Villa, 2010); es decir, una conjunción de motivos, actitudes y valores, conocimientos y habilidades, técnicas, normas y procedimientos que diferencian la actuación o comportamiento del directivo en su desempeño académico-profesional.

En este sentido, para autores como (Hauge, Norenes y Vedøy, 2014; Ottestad, 2013) asistimos a una cultura escolar en la que el líder de la escuela promueve las TIC para planificar, desarrollar y compartir la práctica docente, donde predominan las pedagogías centradas en los estudiantes y las acciones de liderazgo que fomentan la competencia digital entre el personal.

Es por ello que, en los últimos años, se ha pasado de un interés centrado en el liderazgo de la dirección a un liderazgo de amplio espectro en donde las actuaciones se centran en la totalidad de la comunidad educativa. Se habla así del liderazgo distribuido, de carácter compartido, facilitador, integrador, motivador y creador (Avolio, Walumbwa, y Weber, 2009; Fernández, 2007; Ilies, Judge y Wagner, 2006; Kocolowski, 2010; Printy, Marks y Bowers, 2010) fomentando así que el profesorado se implique en estimular un clima que facilite la autonomía en el aprendizaje de los alumnos, lo que redundará en una mejora de los resultados y de los aspectos organizativos del centro educativo.

Con atención a estas tendencias, este artículo analiza la relación entre la distribución del liderazgo educativo de los equipos directivos y de los órganos de coordinación docentes de los IES de Melilla y las TIC como herramientas que deben implementar dicho liderazgo en los ámbitos pedagógico y organizativo (Gewerc y Montero, 2013; GutiérrezSantiuste y Gallego-Arrufat, 2013). Ello permite estudiar dimensiones de la distribución del liderazgo como la comunicación, la colaboración, la confianza, el compromiso, la gestión, el cambio cultural, la innovación y la integración TIC en los procesos de enseñanza aprendizaje (Mwawasi, 2014).

En concordancia, el artículo plantea los siguientes objetivos:

1. Analizar la percepción que poseen los equipos directivos, coordinadores de proyectos educativos y jefes de departamento de los IES de la ciudad autónoma de Melilla sobre el liderazgo distribuido en sus centros.

2. Analizar la percepción que poseen los equipos directivos, coordinadores de proyectos educativos y jefes de departamento de los IES de la ciudad autónoma de Melilla sobre el empleo de las TIC para favorecer el liderazgo distribuido.

\section{METODOLOGÍA}

\subsection{Instrumento}

Para llevar a cabo la investigación y alcanzar los objetivos, se ha elaborado ad hoc un cuestionario con 35 ítems, tipo escala ordinal de cuatro valores (1 = Nada, $2=$ Algo, 3 = Bastante, $4=$ Mucho). Estos ítems se distribuyen en tres dimensiones: Datos académicos y personales (DAP) Liderazgo Distribuido (LD) en los IES de Melilla y Empleo de las TIC para favorecer el Liderazgo Distribuido (TIC). Se ha elegido una escala para evitar las respuestas neutras y lograr un posicionamiento contundente de los participantes sobre las temáticas en cuestión. 
Tabla 1: Dimensiones, subdimensiones e ítems del cuestionario

\begin{tabular}{c|l|c}
\hline Dimensiones & \multicolumn{1}{c}{ Subdimensiones } & \multicolumn{1}{c}{ Ítems } \\
\hline Datos Académicos y & Datos personales 1, 2, 3, 4 \\
Personales (DAP) & Datos académicos 5, 6, 7, 8 \\
\hline & Colaboración & 9,10 \\
& Confianza & 11,12 \\
Liderazgo & Compromiso & 13,14 \\
Distribuido en los & Gestión & $15,16,17,18$ \\
IES de Melilla (LD) & Calidad educativa & $19,20,21$ \\
& Comunicación & 22,23 \\
& & \\
\hline Empleo de las TIC & Innovación & $24,25,26,27$, \\
para favorecer el & & 28 \\
Liderazgo & Herramientas web 2.0 & $29,30,31,32$ \\
Distribuido (TIC) & PLE & $33,34,35$ \\
\hline
\end{tabular}

Los datos personales incluyen un código de identificación, nombre del instituto, sexo, edad. Los académicos incluyen años de experiencia, cargo de responsabilidad en el instituto, especialidad en su formación y grado académico.

Los ítems de las dimensiones Liderazgo distribuido en los institutos de educación secundaria de Melilla y Empleo de las TIC para favorecer el Liderazgo distribuido, junto con sus correspondientes siglas, se detallan en la tabla 2.

Tabla 2. Lista de ítems de la dimensión Liderazgo distribuido en los IES de Melilla

\begin{tabular}{ll}
\hline Ítems & Siglas
\end{tabular}

9. El equipo directivo toma decisiones de forma colegiada y consensuada

EDC

10. El equipo directivo favorece la participación de los distintos sectores de la comunidad educativa en la exposición de propuestas para la mejora de la infraestructura y equipamiento del centro

11. El equipo directivo fomenta un ambiente de confianza y de respeto entre todo el personal del centro

12. El equipo directivo inspira profesionalidad a todo el profesorado por su forma de trabajar y abordar los problemas

13. Se implica en el diseño y mejora del PEC

14. El equipo directivo fomenta el compromiso y la concienciación del profesorado en temas de igualdad, salud, seguridad, medio ambiente y entorno

15. El equipo directivo elabora el presupuesto teniendo en cuenta las propuestas y necesidades del profesorado y de los departamentos didácticos

16. Como profesor, cómo calificaría la gestión del equipo directivo

17. Como profesor, cómo calificaría la gestión del JDP jefe de departamento

18. Como profesor, cómo calificaría la gestión de la junta de tutores

19. El equipo directivo reflexiona colectivamente sobre los objetivos y resultados obtenidos por el ERC centro educativo

20. El equipo directivo escoge como colaboradores

a profesores con iniciativa y valía personal

CIP

21. El equipo directivo es accesible y escucha al personal

EDA

22. El equipo directivo promueve una comunicación $\quad$ ECC clara y fluida entre los miembros de la comunidad

Los ítems de la dimensión Empleo de las TIC para favorecer el liderazgo distribuido en los IES de Melilla, y sus correspondientes siglas se detallan en la tabla 3.

Tabla 3. Lista de ítems de la dimensión Empleo de las TIC para favorecer el LD en los IES de Melilla

Ítems

Siglas

23. Se emplean las TIC para distribuir las tareas TED entre todos los miembros del equipo directivo

24. Se emplean las TIC para distribuir las tareas TDP entre todos los miembros del departamento $\quad$ TDP 25. Se emplean las TIC para distribuir las tareas TJT entre todos los miembros de la junta de tutores

26. El equipo directivo promueve la innovación TIC como factor clave de la integración educativa y la acción educativa intercultural

27. Los profesores noveles son más dados a la innovación TIC en el proceso de enseñanza- PNI aprendizaje

28. Las herramientas TIC son útiles para que el equipo directivo coordine todas las actividades del TCA instituto

29. Las herramientas TIC son útiles para que el jefe de departamento fomente la coordinación docente TCD entre todos sus miembros

30. Las herramientas TIC son útiles para que el tutor fomente la participación de sus alumnos en las TFP actividades del instituto

31. Se emplean en su centro las redes sociales para realizar convocatorias oficiales (claustros, comisiones de coordinación, consejo escolar, juntas de tutores)

32. Se emplean los PLE (entornos personales de aprendizaje) para implementar la coordinación con el resto de miembros del equipo directivo y/o del departamento

33. Utiliza los PLE para reflexionar y debatir sobre la gestión y organización de su centro educativo

34. Como docente se beneficia de los PLE para PLG gestionar su propio aprendizaje

35. Considera que la integración de las TIC en el currículo fomenta el éxito escolar del alumnado 


\subsection{Población y muestra}

La población de esta investigación son los miembros de los equipos directivos, jefes de departamento y coordinadores de proyectos educativos de los institutos de enseñanza secundaria (IES) tanto públicos como privados de la Ciudad Autónoma de Melilla.

La totalidad de la población consta de 181 personas $(\mathrm{N}=181)$, pertenecientes a nueve IES de Melilla. El total de personas que participaron en el estudio y contestaron el cuestionario es de 145 (80.11\%).

La tabla 4 ofrece algunos detalles de la muestra.

Tabla 4. Distribución de la población y la muestra por IES

\begin{tabular}{c|c|c|c}
\hline IES & Población & $\begin{array}{c}\mathrm{N}^{\mathbf{0}} \text { de } \\
\text { cuestionarios } \\
\text { recibidos }\end{array}$ & $\begin{array}{c}\% \text { de la } \\
\text { muestra }\end{array}$ \\
\hline $\begin{array}{c}\text { Leopoldo } \\
\text { Queipo }\end{array}$ & 22 & 21 & $95.45 \%$ \\
$\begin{array}{c}\text { Enrique Nieto } \\
\text { Virgen de la }\end{array}$ & 23 & 20 & $86.95 \%$ \\
$\quad$ Victoria & 19 & 11 & $57.89 \%$ \\
$\begin{array}{c}\text { Juan A. } \\
\text { Fernández }\end{array}$ & 23 & 22 & $95.65 \%$ \\
$\begin{array}{c}\text { Miguel } \\
\text { Fernández }\end{array}$ & 26 & 26 & $100 \%$ \\
$\begin{array}{c}\text { Rusadir } \\
\text { Carmen Conde } \\
\text { Abellán }\end{array}$ & 24 & 23 & $95.83 \%$ \\
$\begin{array}{c}\text { La Salle } \\
\text { Buen Consejo }\end{array}$ & 18 & 6 & $75 \%$ \\
\hline$\quad$ TOTAL & 181 & 145 & $44.44 \%$ \\
\hline
\end{tabular}

(1) Centro público de educación de personas adultas

En esta muestra, un $51 \%$ son hombres y un $49 \%$ mujeres. Las personas con edades entre 41 y 50 años son los más numerosas del estudio, representando el $37.9 \%$, seguido de un $27.6 \%$ que se encuentra en el rango de edad entre 31 y 40 años. Finalmente, un $24.8 \%$ de la muestra tiene una edad entre 51 y 60 años.

En función del sexo (SEX) de los participantes encuestados, únicamente aparece una diferencia estadísticamente significativa con la variable (AEC) "años de experiencia en cargos", en donde la representación masculina en el cargo de director es del $66.7 \%$ frente al $33.3 \%$ de femenina. En el caso del cargo de jefe de estudios o similares, encontramos el $58.1 \%$ con más años de experiencia en dichos cargos frente al $41.9 \%$ femenino. Respecto al cargo de secretario toda la representación es masculina. En cuanto al puesto de jefe de departamento existe una mayor representación femenina (56\%). Por último, como coordinador TIC o similar también se da una mayor representación femenina, con el $52.9 \%$.

Atendiendo a los años de experiencia laboral, un $42.8 \%$ son profesores con una experiencia entre los 11 y 20 años. Cerca del $20 \%$ poseen una experiencia de 21 a 30 años, el $18.6 \%$ de uno a diez años de experiencia, el $15.9 \%$ tienen de 21 a 30 años de experiencia y en torno al 3\% más de 40 años.

\subsection{Validez y fiabilidad el instrumento}

Con el objetivo de medir el grado de fiabilidad y consistencia interna del instrumento, se calculó el Alfa de Cronbach (Cronbach, 1951), arrojando un valor de .939 para la dimensión Liderazgo distribuido en los IES de Melilla y de .826 para la dimensión Empleo de las TIC para favorecer el liderazgo distribuido; por lo tanto, el nivel de confiabilidad del instrumento es adecuado (Buendía, 1997; George y Mallery, 2003; Nunnally, Bernstein, y Berge, 1967; Rodríguez, 2011).

Para confirmar la validez del instrumento, este se sometió al juicio de nueve expertos: siete son profesores (todos doctores, tres titulares y cuatro contratados) que ejercen docencia e investigación en universidades españolas (Universidad Autónoma de Madrid, Universidad de Granada, Universidad de Jaén y Universidad de Málaga) y dos son directores (licenciados) de IES en la ciudad de Melilla. Todos son especialistas en el campo de la Organización Escolar y en el uso organizativo de las TIC. En promedio tienen 15.44 años de experiencia laboral.

Las sugerencias cualitativas que los expertos realizaron al instrumento fueron incorporadas al mismo, garantizando la validez del constructo.

\section{RESULTADOS ESTADÍSTICOS}

\subsection{El liderazgo distribuido (LD) en los IES de Melilla}

En la tabla 5 se muestran la distribución de frecuencias, y otros estadísticos, de las variables de la dimensión Liderazgo distribuido en los IES de Melilla, según la percepción de los equipos directivos, coordinadores de proyectos educativos y jefes de departamento. 
Tabla 5. Liderazgo distribuido en los IES de Melilla: frecuencias, medias, desviación estándar, mediana y rango intercuartílico

\begin{tabular}{|c|c|c|c|c|c|c|c|c|c|c|c|c|c|c|}
\hline \multirow{2}{*}{ Ítems } & \multicolumn{2}{|c|}{ Nada } & \multicolumn{2}{|c|}{ Algo } & \multicolumn{2}{|c|}{ Bastante } & \multicolumn{2}{|c|}{ Mucho } & \multicolumn{4}{|c|}{ Análisis de medias } & \multicolumn{2}{|c|}{ Total } \\
\hline & $\mathrm{N}$ & $\%$ & $\mathrm{~N}$ & $\%$ & $\mathrm{~N}$ & $\%$ & $\mathrm{~N}$ & $\%$ & $\mathrm{M}$ & $\sigma_{\mathrm{X}}$ & $\mathrm{Me}$ & IQR & $\mathrm{N}$ & $\%$ \\
\hline EDC & 7 & 4.8 & 24 & 16.6 & 73 & 50.3 & 41 & 28.3 & 3.02 & .803 & 3 & 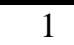 & 145 & 100 \\
\hline EDP & 7 & 4.8 & 28 & 19.3 & 70 & 48.3 & 40 & 27.6 & 2.99 & .816 & 3 & 1 & 145 & 100 \\
\hline EDR & 10 & 6.9 & 18 & 12.4 & 55 & 37.9 & 62 & 42.8 & 3.17 & .898 & 3 & 1 & 145 & 100 \\
\hline EDT & 10 & 6.9 & 31 & 21.4 & 59 & 40.7 & 45 & 31.0 & 2.96 & .897 & 3 & 2 & 145 & 100 \\
\hline PEC & 7 & 4.8 & 23 & 15.9 & 64 & 44.1 & 51 & 35.2 & 3.10 & .83 & 3 & 1 & 145 & 100 \\
\hline EDE & 7 & 4.8 & 29 & 20.0 & 66 & 45.5 & 43 & 29.7 & 3.00 & .833 & 3 & 2 & 145 & 100 \\
\hline EPN & 15 & 10.3 & 26 & 17.9 & 72 & 49.7 & 32 & 22.1 & 2.83 & .890 & 3 & 1 & 145 & 100 \\
\hline EDI & 7 & 4.8 & 26 & 17.9 & 70 & 48.3 & 42 & 29.0 & 3.01 & .816 & 3 & 1 & 145 & 100 \\
\hline JDP & 2 & 1 & 28 & 19.3 & 89 & 61.4 & 26 & 17.9 & 2.96 & .655 & 3 & $\mathbf{0}$ & 145 & 100 \\
\hline JTT & 8 & 5.5 & 40 & 27.6 & 73 & 50.3 & 24 & 16.6 & 2.78 & .786 & 3 & 1 & 145 & 100 \\
\hline ERC & 13 & 9.0 & 11 & 7.6 & 69 & 47.6 & 52 & 35.9 & 3.10 & .888 & 3 & 1 & 145 & 100 \\
\hline CIP & 10 & 6.9 & 27 & 18.6 & 52 & 35.9 & 56 & 38.6 & 3.06 & .922 & 3 & 2 & 145 & 100 \\
\hline EDA & 5 & 3.4 & 20 & 13.8 & 47 & 32.4 & 73 & 50.3 & 3.30 & .834 & 4 & & 145 & 100 \\
\hline ECC & 9 & 6.2 & 31 & 21.4 & 57 & 39.3 & 48 & 33.1 & 2.99 & .894 & 3 & 2 & 145 & 100 \\
\hline
\end{tabular}

Se observa que en todas las variables de la dimensión Liderazgo distribuido en los IES de Melilla, hay una mediana mayor o igual a $3 \mathrm{y}$ promedios mayores a 2,78; esto indica una percepción cercana a la categoría Bastante, para todas las variables. La variable EDA es la que muestra el mayor promedio (3.30) y la mayor media (4). Las desviaciones estándar tienen valores entre .655 (la variable JDP) y .922 (la variable CIP), lo que señala que no hay uniformidad en cuanto la distancia de los datos respecto a su promedio. La mayoría de las variables, como EDC, EDP, EDR, PEC, EPN, EDI, JTT, ERC y EDA tienen un rango intercuartílico igual a 1 , lo que indica una dispersión relativamente baja. El menor rango intercuartílico lo presenta JDP, el cual es cero. Las variables EDT, EDE, CIP y ECC presentan dispersiones relativamente altas, con un rango intercuartílico igual a 2 .

Una vez cruzados los datos entre la variable "centro educativo" (IES) y los ítems de la dimensión Liderazgo distribuido en los IES de Melilla, observamos que en cuanto a "la toma de decisiones de forma colegiada y consensuada por parte del equipo directivo" (EDC), llama la atención que el $21.7 \%$ de los profesores participantes del IES Rusadir considera que la toma de decisiones no se hace de forma colegiada. En referencia a si "el equipo directivo de los institutos favorece la participación de los distintos sectores de la comunidad educativa...", más del 50\% del profesorado de centros como Leopoldo Queipo, Enrique Nieto, Juan A. Fernández Pérez y Miguel Fernández afirma positivamente, lo que constituye el $48.3 \%$ de los docentes participantes. En relación a si "el equipo directivo fomenta un ambiente de confianza y de respeto entre todo el personal del centro" (EDR), se aprecia que en el IES Leopoldo Queipo el $80.9 \%$ de los docentes lo valoran positivamente; así como el $85 \%$ de los docentes del centro Enrique Nieto y el $90 \%$ de los pertenecientes al IES Virgen de la Victoria. Destaca negativamente el 30\% de los docentes del IES Juan. A. Fernández Pérez y el $30.4 \%$ de los docentes del centro Rusadir. En cuanto a si "el equipo directivo inspira profesionalidad a todo el profesorado por su forma de trabajar y abordar los problemas" (EDT), se observa a nivel general que el $71.7 \%$ del total de docentes que participan en el estudio lo perciben positivamente. Los resultados relativos a si "el equipo directivo escoge a los colaboradores según su iniciativa y valía personal" (CIP), el $80 \%$ de los docentes del IES Enrique Nieto y el $82 \%$ d los encuestados del IES Virgen de la Victoria lo perciben de forma positiva. En general todos los centros se asemejan en cuanto a la percepción de su profesorado, excepto el IES Rusadir que lo valora con un $60 \%$ negativamente.

Finalmente, en cuanto a si "el equipo directivo promueve una comunicación fluida y clara con todos los miembros de la comunidad" (ECC), por centros, la mayoría de los docentes tienen una visión positiva al respecto, superando el $60 \%$ de cada uno de los institutos entre los grados Bastante y Mucho. Cabría destacar en un nivel Nada el $55.6 \%$ de representación del IES Rusadir y el $19.4 \%$ en la categoría Algo, porcentajes representativos en comparación con los demás centros. 


\subsection{Factores que explican la dimensión liderazgo distribuido (LD) en los IES de Melilla}

Como resultado de realizar el test de esfericidad de Barlett sobre los datos, se ha obtenido un valor de chi-cuadrado aproximado de 1330.014 (con un grado de libertad de $91 \mathrm{y}$ una significancia asintótica menor a .0001 y un índice de KMO de .939) Estos resultados han llevado a efectuar el análisis factorial, escogiendo el método de Análisis de componentes principales (ACP), rotando las variables a través del procedimiento Varimax (Kaiser, 1958). Se ha elegido este análisis factorial, porque permite reducir el conjunto de variables de la dimensión a un número menor de factores que lo representa, y esto facilita su interpretación. Asimismo, se aclara que el presente estudio busca un modelo minimalista y sintético de las dimensiones; desde esta perspectiva, lo que se busca es encontrar los dos o tres factores con más poder explicativo, que aborden aproximadamente un $60 \%$ de la explicación de la varianza.

Seguidamente, se muestra la tabla de varianza total explicada (tabla 6) en la que, siguiendo el método de extracción de componentes principales, queda explicada la variabilidad total por cada componente. Son dos los factores que explican la primera dimensión del instrumento utilizado. Con un total de un $63.73 \%$, superior al $60 \%$ que se busca.

Tabla 6. Porcentaje de varianza del Liderazgo distribuido en los IES de Melilla, explicado por autovalores iniciales

\begin{tabular}{|c|c|c|c|c|c|c|c|c|c|}
\hline \multirow[t]{2}{*}{ Componente } & \multicolumn{3}{|c|}{$\begin{array}{c}\text { Autovalores iniciales (análisis } \\
\text { factorial ACP, rotación Varimax) }\end{array}$} & \multicolumn{3}{|c|}{$\begin{array}{l}\text { Sumas de las saturaciones al } \\
\text { cuadrado de la extracción }\end{array}$} & \multicolumn{3}{|c|}{$\begin{array}{l}\text { Suma de las saturaciones al } \\
\text { cuadrado de la rotación }\end{array}$} \\
\hline & Total & $\begin{array}{l}\% \text { de la } \\
\text { varianza }\end{array}$ & $\%$ acumulado & Total & $\begin{array}{c}\% \text { de la } \\
\text { varianza }\end{array}$ & $\%$ acumulado & Total & $\begin{array}{c}\% \text { de la } \\
\text { varianza }\end{array}$ & $\begin{array}{c}\% \\
\text { acumulado }\end{array}$ \\
\hline 1 & 7.894 & 56.387 & 56.387 & 7.894 & 56.387 & 56.387 & 6.581 & 47.007 & 47.007 \\
\hline 2 & 1.029 & 7.346 & 63.733 & 1.029 & 7.346 & 63.733 & 2.342 & 16.727 & 63.733 \\
\hline 3 & .808 & 5.774 & 69.508 & & & & & & \\
\hline 4 & .733 & 5.234 & 74.741 & & & & & & \\
\hline 5 & .584 & 4.175 & 78.916 & & & & & & \\
\hline 6 & .550 & 3.930 & 82.846 & & & & & & \\
\hline 7 & .493 & 3.522 & 86.368 & & & & & & \\
\hline 8 & .377 & 2.691 & 89.059 & & & & & & \\
\hline 9 & .345 & 2.461 & 91.520 & & & & & & \\
\hline 10 & .317 & 2.261 & 93.781 & & & & & & \\
\hline 11 & .253 & 1.809 & 95.590 & & & & & & \\
\hline 12 & .233 & 1.666 & 97.256 & & & & & & \\
\hline 13 & .212 & 1.517 & 98.773 & & & & & & \\
\hline 14 & .172 & 1.227 & 100.000 & & & & & & \\
\hline
\end{tabular}

Se puede deducir que los equipos directivos, los jefes de departamentos y los coordinadores de proyectos educativos de los IES de Melilla, opinan que la dimensión Liderazgo distribuido en los IES de Melilla se compone de dos factores:

1. Gestión, organización y participación del equipo directivo del centro que se compone de las variables EDC, EDR, EDT, PEC, EPN, ERC, CIP, EDA, ECC que se concretan en las valoraciones que le dan los encuestados a subdimensiones como la confianza (EDR, EDT), la comunicación (EDA, ECC), la calidad educativa (ECR, CIP) o la colaboración (EDC) para determinar la incidencia del LD en los órganos con responsabilidad organizativa y de gobierno en los IES de Melilla.
2. Percepciones del profesorado sobre la gestión de equipo directivo y encargados de la coordinación docente. Incluye así las variables EDI, JDP y JTT que hacen referencia a la percepción del profesorado sobre la gestión del equipo directivo, jefe de departamento y junta de tutores.

En la tabla 7 se detallan los resultados del análisis factorial, indicando lo que cada variable aporta al factor. Por tanto, cada factor se formará a partir todas aquellas variables que mayor aportación tengan hacia él, considerando todas aquellas que aporten más de $0.4( \pm 0.4)$. 
Tabla 7. Matriz de rotación final de los ítems de la dimensión Liderazgo distribuido en los IES de Melilla

\begin{tabular}{c|c|c|c|c|c}
\hline \multicolumn{6}{c}{ Matriz de componentes rotados } \\
\hline Ítems & 1 & 2 & Ítems & 1 & 2 \\
\hline EDC & $\mathbf{. 7 7 8}$ & .079 & EDI & $\mathbf{. 4 3 4}$ & $\mathbf{. 7 6 2}$ \\
EDP & .386 & .101 & JDP & .082 & $\mathbf{. 8 2 0}$ \\
EDR & $\mathbf{. 7 8 1}$ & .267 & JTT & .261 & $\mathbf{. 7 1 1}$ \\
EDT & $\mathbf{. 8 2 2}$ & .257 & ERC & $\mathbf{. 6 4 6}$ & $\mathbf{. 4 0 8}$ \\
PEC & $\mathbf{. 6 8 4}$ & .384 & CIP & $\mathbf{. 6 9 7}$ & .349 \\
EDE & .393 & .280 & EDA & $\mathbf{. 6 7 5}$ & $\mathbf{. 4 4 5}$ \\
EPN & $\mathbf{. 7 5 7}$ & .075 & ECC & $\mathbf{. 7 3 5}$ & .322 \\
\hline
\end{tabular}

\subsection{El empleo de las TIC para favorecer el liderazgo distribuido en los IES de Melilla}

En la tabla 8 se muestran la distribución de frecuencias, y otros estadísticos, de las variables que componen la dimensión Empleo de las TIC para favorecer el liderazgo distribuido, según la percepción de los equipos directivos, coordinadores de proyectos educativos y jefes de departamento.

Tabla 8. Empleo de las TIC para favorecer el liderazgo distribuido: frecuencias, medias, desviación estándar, mediana y rango intercuartílico

\begin{tabular}{|c|c|c|c|c|c|c|c|c|c|c|c|c|c|c|}
\hline \multirow{2}{*}{ Ítems } & \multicolumn{2}{|c|}{ Nada } & \multicolumn{2}{|c|}{ Algo } & \multicolumn{2}{|c|}{ Bastante } & \multicolumn{2}{|c|}{ Mucho } & \multicolumn{4}{|c|}{ Análisis de medias } & \multicolumn{2}{|c|}{ Total } \\
\hline & $\mathrm{N}$ & $\%$ & $\mathrm{~N}$ & $\%$ & $\mathrm{~N}$ & $\%$ & $\mathrm{~N}$ & $\%$ & M & $\sigma_{\mathrm{X}}$ & $\mathrm{Me}$ & IQR & $\mathrm{N}$ & $\%$ \\
\hline TED & 12 & 8.3 & 32 & 22.1 & 58 & 40.0 & 43 & 29.7 & 2.91 & .920 & 3 & 2 & 145 & 100 \\
\hline TDP & 11 & 7.6 & 34 & 23.4 & 68 & 46.9 & 32 & 22.1 & 2.83 & .858 & 3 & 1 & 145 & 100 \\
\hline TJT & 13 & 9.0 & 52 & 35.9 & 60 & 41.4 & 20 & 13.8 & 2.60 & .837 & & 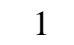 & 145 & 100 \\
\hline TII & 8 & 5.5 & 44 & 30.3 & 55 & 37.9 & 38 & 26.2 & 2.85 & .877 & 3 & 2 & 145 & 100 \\
\hline PNI & 6 & 4.1 & 43 & 29.7 & 61 & 42.1 & 35 & 24.1 & 2.86 & .830 & 3 & 1 & 145 & 100 \\
\hline TCA & 7 & 4.8 & 28 & 19.3 & 66 & 45.5 & 44 & 30.3 & 3.03 & .845 & 3 & 1 & 145 & 100 \\
\hline TCD & 8 & 5.5 & 30 & 20.7 & 70 & 48.3 & 37 & 25.5 & 2.94 & .827 & 3 & 2 & 145 & 100 \\
\hline TFP & 10 & 6.9 & 39 & 26.9 & 65 & 44.8 & 31 & 21.4 & 2.81 & .852 & 2 & 1 & 145 & 100 \\
\hline RSC & 50 & 34.5 & 33 & 22.8 & 41 & 28.3 & 21 & 14.5 & 2.23 & 1.078 & 2 & 2 & 145 & 100 \\
\hline PLE & 34 & 23.4 & 60 & 41.4 & 44 & 30.3 & 7 & 4.8 & 2.17 & .842 & 0 & 1 & 145 & 100 \\
\hline PLR & 36 & 24.8 & 62 & 42.8 & 38 & 26.2 & 9 & 6.2 & 2.14 & .863 & 2 & 2 & 145 & 100 \\
\hline PLG & 29 & 20.0 & 59 & 40.7 & 44 & 30.3 & 13 & 9.0 & 2.28 & .887 & 2 & 1 & 145 & 100 \\
\hline TFE & 7 & 4.8 & 41 & 28.3 & 65 & 44.8 & 32 & 22.1 & 2.84 & .822 & 3 & 1 & 145 & 100 \\
\hline
\end{tabular}

Se observa que en la mayoría de las variables de la dimensión Empleo de las TIC para favorecer el liderazgo distribuido, hay una mediana mayor o igual a 3 y promedios mayores a 2,60; esto indica una percepción cercana a la categoría Bastante, para todas estas variables.

Sin embargo, hay cuatro variables de esta dimensión, entre ellas RSC, PLE, PLR y PLG, que tienen medianas iguales a 2 y promedios con valores entre 2.14 y 2.28 . Esto indica una percepción de los sujetos participantes, con menor intensidad que las anteriores hacia estas variables, más cercanos a la categoría Bastante.

La variable TCA es la que muestra el mayor promedio (3.03). Las desviaciones estándar tienen valores entre .822 (la variable TFE) y 1.078 (la variable CIP), lo que señala distancias medianas y distancias altas de los datos respecto a sus respectivos promedios. Hay un subgrupo de ocho variables con un rango intercuartílico igual a 1 , es decir con una dispersión relativamente baja; y hay otro subgrupo de cinco variables con un rango intercuartílico igual a 2, es decir, con una dispersión relativamente alta.

A continuación, mostramos los cruces que presentan una diferencia estadísticamente significativa entre la variable "centro educativo" (IES) y las variables correspondientes a la dimensión Tecnologías de la Comunicación e Innovación (TIC).

En cuanto al "empleo de las TIC para la distribución de las tareas docentes entre los miembros de la Junta de Tutores" (TJT), los institutos Leopoldo Queipo y Juan A. Fernández Pérez obtienen una mayor representación docente en el empleo de las TIC. En relación a "la promoción de la innovación TIC de los equipos directivos para la integración educativa y la acción educativa intercultural" (TII) es un aspecto valorado positivamente por los docentes de los IES Enrique Nieto y Leopoldo Queipo. En referencia a si "los profesores noveles tienen más tendencia a innovar con TIC en el proceso de enseñanza-aprendizaje" (PNI), las apreciaciones del profesorado se reparten 
a favor y en contra de forma equitativa en el IES Leopoldo Queipo, dato que contrasta con el $80 \%$ del profesorado del IES Enrique Nieto que lo valora positivamente (Bastante y Mucho), datos ligeramente superiores se recogen en el IES Virgen de la Victoria con Bastante (63.6\%) y Mucho (27.3\%). En cuanto al "empleo de los PLE (Entornos Personales de Aprendizaje) para la implementación de la coordinación docente con el resto de miembros del equipo directivo y /o departamento" destaca que los emplea el 35\% de los docentes del IES Enrique Nieto y el $45.5 \%$ del profesorado perteneciente al IES Juan A. Fernández Pérez en el rango de Bastante. En relación a si "la integración de las TIC en el currículo fomenta el éxito escolar del alumno", el profesorado de los IES Enrique Nieto (45\%), Juan A. Fernández Pérez (54.5\%), Miguel Fernández (45.7\%), La Salle (50\%) y Rusadir (47.8\%) lo valoran positivamente. Por el contrario, el $75 \%$ de los docentes del centro Buen Consejo lo valora de forma negativa.

\subsection{Factores que explican el empleo de las TIC para favorecer el liderazgo distribuido en los IES de Melilla}

Siguiendo las mismas directrices que en el apartado anterior, se interpretan los resultados a través los valores obtenidos en las pruebas de esfericidad de Barlett. En este caso se muestra un valor de chicuadrado de 724.963 (con 78 grados de libertad, una significancia asintótica menor a .0001 , y un valor de KMO igual a .783). A partir de estos resultados se decide hacer el correspondiente análisis factorial mediante el método PCA con rotación Varimax de Káiser.

En la tabla 9 siguiente, se observa la variabilidad total por cada componente y el porcentaje que explica cada uno. Tres variables explican la segunda dimensión del cuestionario aplicado, con un porcentaje explicativo del $\mathbf{5 9 . 6 2 \%}$, suficientemente cercano al $60 \%$ que se ha planteado.

Tabla 9. Porcentaje de varianza del Empleo de las TIC para favorecer el liderazgo distribuido, en los IES de Melilla, explicado por autovalores iniciales

\begin{tabular}{|c|c|c|c|c|c|c|c|c|c|}
\hline \multirow[t]{2}{*}{ Componente } & \multicolumn{3}{|c|}{ Autovalores iniciales } & \multicolumn{3}{|c|}{$\begin{array}{l}\text { Sumas de las saturaciones al } \\
\text { cuadrado de la extracción }\end{array}$} & \multicolumn{3}{|c|}{$\begin{array}{l}\text { Suma de las saturaciones al } \\
\text { cuadrado de la rotación }\end{array}$} \\
\hline & Total & $\begin{array}{c}\% \text { de la } \\
\text { varianza }\end{array}$ & $\%$ acumulado & Total & $\begin{array}{c}\% \text { de la } \\
\text { varianza }\end{array}$ & $\%$ acumulado & Total & $\begin{array}{c}\% \text { de la } \\
\text { varianza }\end{array}$ & $\%$ acumulado \\
\hline 1 & 4.358 & 33.527 & 33.527 & 4.358 & 33.527 & 33.527 & 2.817 & 21.669 & 21.669 \\
\hline 2 & 2.230 & 17.155 & 50.682 & 2.230 & 17.155 & 50.682 & 2.760 & 21.231 & 42.900 \\
\hline 3 & 1.162 & 8.936 & 59.618 & 1.162 & 8.936 & 59.618 & 2.173 & 16.718 & 59.618 \\
\hline 4 & .950 & 7.311 & 66.929 & & & & & & \\
\hline 5 & .786 & 6.048 & 72.977 & & & & & & \\
\hline 6 & .699 & 5.374 & 78.351 & & & & & & \\
\hline 7 & .661 & 5.082 & 83.432 & & & & & & \\
\hline 8 & .537 & 4.129 & 87.561 & & & & & & \\
\hline 9 & .497 & 3.823 & 91.384 & & & & & & \\
\hline 10 & .410 & 3.157 & 94.541 & & & & & & \\
\hline 11 & .293 & 2.251 & 96.792 & & & & & & \\
\hline 12 & .223 & 1.716 & 98.508 & & & & & & \\
\hline 13 & .194 & 1.492 & 100.000 & & & & & & \\
\hline
\end{tabular}

Tabla 10. Matriz de rotación final de los ítems de la dimensión Empleo de las TIC para favorecer el liderazgo distribuido

\begin{tabular}{c|c|c|c|c|c|c|c}
\hline \multicolumn{7}{c}{ Matriz de componentes rotados } \\
\hline Ittems & 1 & 2 & 3 & Ítems & 1 & 2 & 3 \\
\hline TED & .104 & $\mathbf{. 8 1 0}$ & .149 & TFP & .203 & .044 & $\mathbf{. 7 6 6}$ \\
TDP & .155 & $\mathbf{. 7 6 1}$ & .213 & RSC & $\mathbf{. 6 6 5}$ & .115 & .002 \\
TJT & .285 & $\mathbf{. 6 9 1}$ & .156 & PLE & $\mathbf{. 8 8 6}$ & .140 & .005 \\
TII & .132 & $\mathbf{. 7 1 3}$ & .176 & PLR & $\mathbf{. 8 4 9}$ & .226 & .085 \\
PNI & .143 & .377 & .347 & PLG & $\mathbf{. 8 1 7}$ & .041 & .169 \\
TCA & .002 & $\mathbf{. 4 7 2}$ & $\mathbf{. 6 5 2}$ & TFE & .065 & .130 & .366 \\
TCD & .019 & .265 & $\mathbf{. 7 6 5}$ & & & & \\
\hline
\end{tabular}

De este análisis deducimos que los equipos directivos, los jefes de departamentos y los coordinadores de proyectos educativos de los IES de Melilla, opinan que el empleo de las TIC para favorecer el liderazgo distribuido se compone de tres factores:

1. Uso de la TIC para la organización de los órganos de gobierno y coordinación docente del centro: En donde las percepciones del profesorado se decantan acerca de la utilización de las TIC ya sea para distribuir las funciones entre los miembros del equipo directivo (TED), los miembros de los 
departamentos (TDP) o los propios tutores (TJT), o bien como recurso que implementa, desde el punto de vista de los encuestados, la integración educativa de los alumnos (TII).

2. Utilidad de las TIC para la coordinación y participación de profesorado, equipo directivo y alumnado: En las percepciones obtenidas por los encuestados, las TIC son herramientas que propician la coordinación por parte del equipo directivo de todas las actividades del centro (TCA), así como la coordinación docente entre los miembros de los distintos departamentos (TCD) y el fomento de la participación de los alumnos en las actividades que se diseñen desde el centro (TFP).

3. Integración y uso de los PLE para la gestión, organización y reflexión del claustro y equipo directivo del centro: Donde se aborda el uso de las redes sociales para las convocatorias oficiales de los órganos de gobierno y de coordinación docente (RSC). Se emplean los entornos personajes de aprendizaje tanto para fomentar la coordinación entre los miembros del equipo directivo y de los departamentos como para establecer un espacio de debate y de reflexión sobre la organización del centro (PLE), (PLR), al tiempo que desde el punto de vista individual los docentes gestionan su propio aprendizaje (PLG).

\section{DISCUSIÓN}

La gestión de los equipos directivos de los institutos de la Ciudad Autónoma de Melilla es, en general, bien valorada por sus docentes; a excepción de los docentes del IES Rusadir. En cuanto a la gestión de los jefes de departamento de dichos centros, la percepción es en general positiva, si bien en los IES Miguel Fernández y Rusadir se percibe mayoritariamente de forma negativa.

Del análisis de las subdimensiones como la confianza (EDR, EDT), la comunicación (EDA, ECC), la calidad educativa (ERC, CIP) o la colaboración (EDC) se deduce que la distribución del liderazgo de los órganos con responsabilidad organizativa y de gobierno en los IES de Melilla es positiva en todos los centros públicos y muy positiva en los dos centros concertados: La Salle y El Buen Consejo. Sin embargo, en el IES Rusadir se deberían evaluar las causas que han llevado al divorcio entre el equipo directivo y el resto del profesorado con el fin de proponer soluciones que redunden en una mejora, en este orden, de: la confianza, la comunicación, el compromiso, la colaboración para generar un grado de gestión positivo que redundaría irremediablemente en una mejora de la calidad educativa del centro.

En el empleo de las TIC en los IES de Melilla, los participantes valoran el carácter integrador y de compensación de desigualdades que presenta para los alumnos. Sin embargo, se precisa de forma urgente que se tomen medidas encaminadas al empleo de las TIC por parte del profesorado tanto a nivel curricular como de trabajo en el aula y de coordinación a nivel horizontal y vertical. En este sentido, es necesaria una oferta de cursos de formación específicos, que se impartan en los mismos centros educativos, orientados al uso y manejo de las TIC para fomentar el liderazgo distribuido.

\section{CONCLUSIONES Y RECOMENDACIONES}

A partir del estudio realizado se ofrecen las siguientes conclusiones y recomendaciones:

1. En general, existe distribución de liderazgo entre los equipos directivos y los órganos de coordinación didáctica de los institutos de la Ciudad Autónoma de Melilla, esta distribución se logra mediante la colaboración entre todo el personal de cada uno de los centros, al tiempo que se fomenta un clima positivo de participación, comunicación y confianza que motiva una mejora de la organización y de la gestión a nivel académico, social e integrador.

2. En algunos IES, el uso de las TIC no fomenta adecuadamente la distribución del liderazgo de los equipos directivos y de los órganos de coordinación didáctica.

3. Se recomienda investigar más profundamente el empleo de las TIC en los IES de Melilla, para lograr esta profundidad se recomiendan estudios cualitativos.

4. Se recomienda llevar a cabo procesos de formación en los IES de Melilla que fomenten el uso eficiente de las TIC, particularmente en lo que respecta a la distribución del liderazgo.

\section{REFERENCIAS}

Avolio, B. J., Walumbwa, F. O., y Weber, T. J. (2009). Leadership: Current theories, research, and future directions. Annual review of psychology, 60, 421-449.

Blau, I., y Presser, O. (2013). E-Leadership of school principals: Increasing school effectiveness by a school data management system. British Journal of Educational Technology, 44(6), 1000-1011.

Buendía, L. (1997). La investigación por encuesta. La investigación observacional. L. Buendía, P. Colás y F. 
Hernández (Coords.), Métodos de investigación en psicopedagogía. Madrid: McGraw-Hill.

Carreño, I. del V. G. (2010). Liderazgo distribuido, una visión innovadora de la dirección escolar: una perspectiva teórica. Omnia, 16(3), 19-36.

Cox, M. J. (2013). Formal to informal learning with IT: research challenges and issues for e-learning. Journal of computer assisted learning, 29(1), 85-105.

Cronbach, L. J. (1951). Coefficient alpha and the internal structure of tests. psychometrika, 16(3), 297-334.

Dexter, S. (2011). School technology leadership: Artifacts in systems of practice. Journal of School Leadership, $21(2)$.

Fernández, M. J. (2007). La dirección escolar ante los retos del siglo XXI. Participación educativa, (5), 23-38.

George, D., y Mallery, M. (2003). Using SPSS for Windows step by step: a simple guide and reference.

Gewerc, A., y Montero, L. (2013). Culturas, formación y desarrollo profesional. La integración de las TIC en las instituciones educativas: Cultures, training and career development. The integration of ICT in educational institutions. Ministerio de Educación. Recuperado a partir de https://repositorio.minedu.gob.pe/bitstream/handle/

Gutiérrez-Santiuste, E., y Gallego-Arrufat, M. J. (2013). Analizar el liderazgo distribuido en entornos virtuales de formación. Recuperado a partir de http://digibug.ugr.es/handle/10481/36279

Harris, A., y Muijs, D. (2004). Improving schools through teacher leadership. McGraw-Hill Education (UK).

Hauge, T. E., Norenes, S. O., y Vedøy, G. (2014). School leadership and educational change: Tools and practices in shared school leadership development. Journal of Educational Change, 15(4), 357-376.

Ilies, R., Judge, T., y Wagner, D. (2006). Making sense of motivational leadership: The trail from transformational leaders to motivated followers. Journal of Leadership \& Organizational Studies, 13(1), 1-22.

Kaiser, H. F. (1958). The varimax criterion for analytic rotation in factor analysis. Psychometrika, 23(3), 187-200.

Kocolowski, M. D. (2010). Shared leadership: Is it time for a change. Emerging Leadership Journeys, 3(1), 22-32.

MECD. (2013). Ley Orgánica 8/2013, de 9 de diciembre, para la mejora de la calidad educativa. Boletín Oficial del Estado. Consultada en el BOE, 295. Disponible en: www. boe. es/diario_boe/txt. php. Recuperado a partir de www.boe.es/buscar/act.php?id=BOE-A-201312886

MECD. (2014). Real Decreto 894/2014, de 17 de octubre, por el que se desarrollan las características del curso de formación sobre el desarrollo de la función directiva. Consultada en el BOE, 270. Disponible en www.boe.es/diario_boe/txt.php?id=BOE-A-2014-494

Murillo, F. J. (2004). Un marco comprensivo de mejora de la eficacia escolar. Investigación, 9(21), 319-359.

Mwawasi, F. M. (2014). Technology leadership and ICT use: Strategies for Capacity Building for ICT integration. Journal of Learning for Development-JLAD, 1(2).

Nunnally, J. C., Bernstein, I. H., y Berge, J. M. ten. (1967). Psychometric theory (Vol. 226). JSTOR. Recuperado a
http://www.jstor.org/stable/pdf/1175619.pdf

Ottestad, G. (2013). School leadership for ICT and teachers' use of digital tools. Nordic Journal of Digital Literacy, 8(1-2), 107-125.
Printy, S., Marks, H., y Bowers, A. (2010). Integrated leadership: How principals and teachers share transformational and instructional influences. $\mathrm{JSl}_{\mathrm{V}} \mathrm{Vol}$ 19-N5, 19, 504.

Rodríguez, J. M. (2011). Métodos de investigación cualitativa. Revista de Investigación Silogismo, 1(08). Recuperado a partir de http://www.cide.edu.co/ojs/index.php/silogismo/articl e/view/64

Schrum, L., y Levin, B. B. (2012). Evidence-based strategies for leading 21 st century schools. Corwin Press.

Villa, A. (2010). Marco pedagógico de la Universidad de Deusto. Bilbao: University of Deusto.

White, D. A. (2014). Distributed leadership for innovative use of ICT in teaching and learning: Empowerment through culture, capacity and collaboration. University of Waikato. 